\title{
Les effets de la révision constitutionnelle du 30 décembre 2020 sur le droit du travail
}

\section{Chakib Boukli Hacène}

\section{(2) OpenEdition}

1 Journals

\section{Édition électronique}

URL : https://journals.openedition.org/rdctss/705

DOI : $10.4000 /$ rdctss.705

ISSN : 2262-9815

Éditeur

Centre de droit comparé du travail et de la sécurité sociale

\section{Édition imprimée}

Date de publication : 1 avril 2021

Pagination : 130-133

ISSN : 2117-4350

\section{Référence électronique}

Chakib Boukli Hacène, « Les effets de la révision constitutionnelle du 30 décembre 2020 sur le droit du travail », Revue de droit comparé du travail et de la sécurité sociale [En ligne], 1 | 2021, mis en ligne le 01 avril 2021, consulté le 02 avril 2022. URL : http://journals.openedition.org/rdctss/705 ; DOI : https:// doi.org/10.4000/rdctss.705

\section{(c) (i) $\odot$}

Revue de droit comparé du travail et de la sécurité sociale est mise à disposition selon les termes de la Licence Creative Commons Attribution - Pas d'Utilisation Commerciale - Pas de Modification 4.0 International. 


\title{
CHAKIB BOUKLI HACÈNE
}

\author{
UNIVERSITÉ DE SAÏDA
}

\section{LES EFFETS DE LA RÉVISION CONSTITUTIONNELLE DU 30 DÉCEMBRE 2020 SUR LE DROIT DU TRAVAIL}

La Constitution algérienne de 1989, de tendance libérale, vient de connaître une nouvelle révision promulguée le 30 décembre 2020. Selon son préambule, cette révision traduirait les aspirations du peuple à des mutations sociales profondes, pacifiquement exprimées depuis le mouvement populaire du 22 février 2019, visant à édifier « une Algérie nouvelle ». Il est intéressant d'examiner les modifications apportées par cette révision du point de vue de la reconnaissance et de la garantie des droits fondamentaux du droit du travail et de la sécurité sociale, d'autant que les précédentes versions de la Constitution, en rupture avec la Constitution socialiste de 1976, reconnaissaient déjà un grand nombre de droits socioprofessionnels.

Cette dernière mouture de la Constitution, rédigée par un comité d'experts nommé par le Président de la République, se veut plus ambitieuse dans la reconnaissance et la garantie d'un large éventail de droits fondamentaux et de libertés publiques. Elle dispose expressément que les droits fondamentaux et les libertés publiques sont garanties par l'Etat et s'imposent à l'ensemble des pouvoirs et institutions public qui ne peuvent les restreindre qu'au moyen d'une loi, lorsque la Constitution elle-même le prévoit et pour des motifs liés au maintien de l'ordre public, de la sécurité et de la protection des constantes nationales, ainsi que pour la sauvegarde d'autres droits et libertés constitutionnellement protégés. En tout état de cause, ces restrictions ne peuvent porter atteinte à l'essence de ces droits et libertés.

Ainsi, en ce qui concerne les droits fondamentaux relatifs au droit social, la nouvelle Constitution reprend l'essentiel des précédentes versions (I) mais en ajoute de nouveaux (II), dans le but de se rapprocher au plus près des Conventions que l'Organisation internationale du travail (OIT) considère comme fondamentales ${ }^{1}$.

\section{I - LA CONSOLIDATION CONSTITUTIONNELLE DES DROITS À CARACTÈRE SOCIOPROFESSIONNEL}

Le législateur constitutionnel reconduit la totalité des droits et libertés garantis par les précédentes versions de la Constitution. Tout d'abord, il maintient le pouvoir exclusif du Parlement à légiférer dans les domaines des droits fondamentaux des personnes, notamment le régime des libertés publiques et la sauvegarde des libertés individuelles

1 En 1998, la Déclaration relative aux principes et droits fondamentaux reconnait huit Conventions fondamentales : le travail forcé et son abolition (Conventions $n^{\circ} 29$ et 105), la liberté syndicale (Convention ${ }^{\circ} 87$ ), le droit d'organisation et de négociation collective (Convention n98), l'égalité de rémunération (Convention $\mathrm{n}^{\circ} 100$ ), la discrimination (Convention $\mathrm{n}^{\circ} 111$ ), l'âge minimum (Convention $\mathrm{n}^{\circ} 138$ ), et enfin les pires formes de travail des enfants (Convention $\mathrm{n}^{\circ} 182$ ), Voir ilo.org, "Conventions et recommandations ». 
des citoyens, ainsi que des règles générales relatives au droit du travail, à la sécurité sociale et à l'exercice du droit syndical (art. 139). II proclame également la supériorité des Traités et Conventions internationaux ratifiés par le Président de la République, dont ceux émanant de l'OIT, portant sur la loi interne (art. 154).

Le législateur constitutionnel reprend également des principes d'ordre général qui peuvent avoir une application en droit du travail, tels que « la promotion de la justice sociale » (art. 9), " la participation effective de tous les citoyens et citoyennes à la vie politique, économique, sociale et culturelle » (art. 35), «l'égalité et la non-discrimination » (art. 37), "l'inviolabilité de la personne humaine ", "la proscription de toute forme de violence physique ou morale ou d'atteinte à la dignité » et "la répression de la torture, les traitements cruels, inhumains ou dégradants ainsi que la traite des personnes» (art. 39), "l'inviolabilité de la vie privée du citoyen » et «la protection des personnes dans les traitements des données à caractère personnel » (art. 47), ainsi que « la garantie de la liberté du commerce, de l'investissement et d'entreprise » (art. 61) qui serait l'expression du principe de la «liberté du commerce et de l'industrie».

S'agissant par ailleurs des droits fondamentaux relevant du droit constitutionnel du travail et de la sécurité sociale, la nouvelle loi fondamentale reprend à l'identique « le droit à la protection, à la sécurité et à l'hygiène dans le travail », "le droit au repos », "le droit à la sécurité sociale », «l'interdiction du travail des enfants » (art. 66), "le droit à l'enseignement et à la formation professionnelle » (art. 65) et « le droit de grève qui doit s'exercer dans le cadre de la loi [qui]peut en interdire ou en limiter l'exercice dans les domaines de la défense nationale et de sécurité, ou pour tous services ou activités publics d'intérêt vital pour la nation » (art. 70). En outre, la loi proclame que l'Etat maintient son engagement constitutionnel en faveur de la promotion de l'apprentissage, des politique d'aide à la création d'emplois, de la parité entre les hommes et les femmes sur le marché de l'emploi, en faveur de la promotion des femmes aux responsabilités dans les institutions et administrations publiques ainsi qu'au niveau des entreprises » (art. 66 et 68). II est à signaler que la parité entre les hommes et les femmes, et la promotion de ces dernières aux postes de responsabilité au sein des entreprises du secteur privé, n'ont pas fait l'objet d'une loi, alors que ces droits constitutionnels ont été consacrés depuis 2016.

\section{II - LA PROCLAMATION CONSTITUTIONNELLE DE NOUVEAUX DROITS À CARACTÈRE SOCIOPROFESSIONNEL}

En premier lieu, il faut noter que tous les droits - dont ceux relatifs au droit social énoncés par la Constitution révisée sont dorénavant qualifiés de "fondamentaux ». Ils bénéficient de ce fait de la protection constitutionnelle contre toute atteinte ou restriction de la part de l'ensemble des pouvoirs et institutions publics. En outre, l'Etat doit veiller à la mise en œuvre législative de ces droits fondamentaux afin d'assurer leur accessibilité, leur visibilité et stabilité.

Par ailleurs, concernant les principes de portée générale qui peuvent intéresser le droit social, l'actuelle révision constitutionnelle apporte une nouvelle « protection aux femmes contre toutes formes de violence, en tous lieux et en toutes circonstances, dans l'espace public, dans la sphère professionnelle et dans la sphère privé » (art. 40). Cela dénote la volonté du législateur constitutionnel d'accentuer la protection de la femme (la considérant ainsi comme un être vulnérable) contre toute forme de violence. II lui apporte alors une double protection puisque l'article 39 de cette Constitution garantit déjà l'inviolabilité de 
la personne humaine (dont la femme) et proscrit par conséquent toute forme de violence physique et morale, et d'atteinte à la dignité.

Quant aux nouveaux droits fondamentaux relevant du droit du travail et de la sécurité sociale, la Constitution reformule quelques droits déjà proclamés dans les précédentes versions et en ajoute de nouveaux, sans pour autant reproduire la totalité des droits fondamentaux proclamés par l'OIT. Tout d'abord, elle reformule le droit fondamental au travail, exprimé sous l'ancienne version en ces termes : "Tous les citoyens ont droit au travail », qui devient : « Le travail est un droit et un devoir ». On constate que l'Etat ne garantit plus le droit au travail comme une obligation stricte, puisque ce droit est parallèlement devenu un devoir vis-à-vis de la société. Cela s'explique par les prérogatives dévolues à un Etat libéral (comme l'Algérie depuis la promulgation de la Constitution de 1989) qui n'assume qu'en partie des fonctions économiques et ne dispose plus du monopole de la répartition des emplois. Par conséquent, l'Etat n'est pas obligé d'octroyer un emploi à tout citoyen ayant l'âge de travailler; il se cantonne plutôt à protéger le droit au travail en mettant en place des mécanismes institutionnels destinés à favoriser l'accès et la réinsertion des demandeurs d'emplois sur le marché du travail. Ceci devrait néanmoins être compensé par une indemnité pour toute personne n'ayant pas pu jouir de ce droit. Cela n'est aujourd'hui pas le cas puisque seuls les travailleurs ayant perdu leur emploi en raison d'une compression d'effectifs peuvent prétendre à une indemnisation, dans le cadre du régime d'assurance chômage ${ }^{2}$.

D'autre part, le droit syndical est également reformulé, passant d'un « droit reconnu à tous les citoyens " à un "droit reconnu [sans précision du bénéficiaire et dont la loi garantit le libre exercice]. Les opérateurs du secteur économique peuvent se constituer en organisations patronales dans le respect de la loi » (art. 69). Cette nouvelle formulation semble, dans son premier alinéa, conforme à la Convention n87 de l'OlT, bien qu'elle puisse, au vu de son énoncé généraliste, porter à confusion dans la mesure où elle sous-entend que le droit syndical est garanti à tous les citoyens sans exception, contrairement à la législation algérienne en vigueur qui restreint ce droit aux seuls travailleurs et employeurs privés.

En revanche, une réelle avancée réside dans la proclamation, pour la première fois depuis l'indépendance du pays en 1962, de la garantie constitutionnelle du libre exercice du droit syndical. Ainsi les travailleurs algériens jouissent dorénavant d'un droit fondamental qui leur garantit d'exercer leurs prérogatives syndicales en toute liberté, à l'échelle professionnelle, interprofessionnelle et à celle de l'entreprise. Ceci aura certainement une incidence sur la législation relative à l'exercice du droit syndical, ainsi que sur sa pratique au sein de l'entreprise, voire au-delà.

Ce n'est pas le cas du deuxième alinéa de l'article 69 cité ci-dessus, qui use de terminologies inappropriées et dont le contenu manque juridiquement de pertinence. En effet, la notion d'« opérateur du secteur économique » n'appartient ni à la terminologie du régime juridique relatif à l'exercice du droit syndical, ni au régime juridique relatif au droit de constituer des associations, ni même au droit international sur la liberté syndicale. Tous les acteurs publics et privés ayant la qualité d'« opérateur économique » n'ont pas vocation à créer des «organisations patronales». Le droit de constituer des « organisations syndicales » se rattache en droit international comme en droit algérien à la notion d' « employeur », et particulièrement d'employeurs privés. Quant à la notion d'« organisation patronale », elle est imprécise. La législation algérienne (ainsi que la plupart des droits nationaux) utilise le terme d'« organisation syndicale » pour désigner un groupement professionnel destiné 


\section{AlgÉRIE}

à permettre aux travailleurs et aux employeurs de défendre leurs intérêts professionnels. En fin de compte, il aurait fallu se contenter du premier alinéa, en précisant que le droit syndical est reconnu aux travailleurs et aux employeurs privés.

Par ailleurs, la nouvelle modification constitutionnelle proclame que « tout travail mérite salaire » (art. 66) afin de garantir aux travailleurs une rémunération en contrepartie du travail fourni. Cette interdépendance entre le travail et le salaire est le résultat logique du caractère synallagmatique du contrat du travail. Cependant, cette conception propre au droit des obligations est tempérée en droit du travail par l'intervention du législateur, pour tenir compte d'une composition du salaire qui ne se réduit pas à l'analyse contractuelle et prend en considération son caractère alimentaire. Ainsi, le paiement du salaire est légalement garanti dans toute une série de situations où le salarié est pourtant dispensé de l'exécution de la prestation de travail à laquelle l'oblige le contrat de travail ${ }^{3}$. Par conséquent, cette protection constitutionnelle manque de pertinence et d'efficacité juridiques. Dès lors, il aurait été plus judicieux de reproduire la Convention fondamentale internationale $\mathrm{n}^{\circ} 100$ sur l'égalité des rémunérations, qui garantit au travailleur une rémunération suffisante pour assurer à lui-même et à sa famille des conditions de vie dignes, ainsi qu'un salaire minimum fixé par la loi et une égalité de rémunération pour un travail de valeur égale.

Enfin, en matière de travail forcé ou obligatoire, la nouvelle mouture constitutionnelle ne traite curieusement que de l'exception à ces formes interdites de travail, en énonçant que «la loi détermine les conditions de réquisition des personnels pour les besoins d'intérêt général » (art. 66). Là encore, il serait impératif d'ajouter au corpus de l'article 66 de la nouvelle Constitution un alinéa traitant de l'interdiction de toutes formes de travail forcé ou obligatoire. Dans cet élan, il serait souhaitable de garantir aussi aux travailleurs et aux employeurs la participation, par la négociation, à la détermination collective des conditions d'emploi et de travail, en concordance avec la Convention $n^{\circ} 98$ sur le droit d'organisation et de négociation collective.

En conclusion, cette nouvelle version de la Constitution algérienne demeure insuffisante en matière de reconnaissance et de garantie des droits fondamentaux du travail et de la sécurité sociale, et mériterait d'être complétée et perfectionnée en vue d'une meilleure concordance avec les Conventions fondamentales de l'Organisation internationale du travail. II serait également nécessaire d'activer la mise en œuvre législative de ces nouveaux droits sociaux fondamentaux, comme la parité entre les hommes et les femmes, la promotion de la femme aux postes de responsabilité au sein des entreprises privées, ou encore le libre exercice du droit syndical, afin d'assurer une plus grande lisibilité de ces droits et leur applicabilité effective.

3 M. N.-E. Koriche, "Les transformations du droit algérien du travail entre statut et contrat : une déréglementation relative », O. P. U., Alger, 2009, Tome 2, p. 89. 